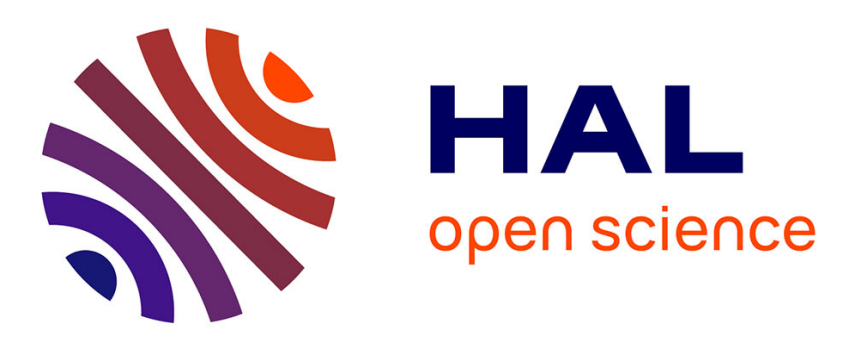

\title{
OBSERVATION OF PENDELLÖSUNG EFFECT IN POLARIZED NEUTRON SCATTERING FROM A MAGNETIC CRYSTAL
}

\author{
J. Baruchel, J. Guigay, C. Mazuré-Espejo, M. Schlenker, J. Schweizer
}

\section{- To cite this version:}

J. Baruchel, J. Guigay, C. Mazuré-Espejo, M. Schlenker, J. Schweizer. OBSERVATION OF PENDELLÖSUNG EFFECT IN POLARIZED NEUTRON SCATTERING FROM A MAGNETIC CRYS-

TAL. Journal de Physique Colloques, 1982, 43 (C7), pp.C7-101-C7-106. 10.1051/jphyscol:1982715 . jpa-00222322

\section{HAL Id: jpa-00222322 https://hal.science/jpa-00222322}

Submitted on 1 Jan 1982

HAL is a multi-disciplinary open access archive for the deposit and dissemination of scientific research documents, whether they are published or not. The documents may come from teaching and research institutions in France or abroad, or from public or private research centers.
L'archive ouverte pluridisciplinaire HAL, est destinée au dépôt et à la diffusion de documents scientifiques de niveau recherche, publiés ou non, émanant des établissements d'enseignement et de recherche français ou étrangers, des laboratoires publics ou privés. 
JOURNAL DE PHYSIQUE

Colzoque C7, supplément au $n^{\circ} 12$, Tome 43, décembre 1982

page $\mathrm{C7}-101$

\author{
OBSERVATION OF PENDELLÖSUNG EFFECT IN POLARIZED NEUTRON SCATTERING \\ FROM A MAGNETIC CRYSTAL \\ J. Baruche1, J.P. Guigay* ${ }^{*}$, C. Mazurë-Espejo ${ }^{+}$, M. Schlenker and J. Schweizer*,** \\ Laboratoire Louis Néel, CNRS-USMG, $166 \mathrm{X}, 38042$ Grenoble Cedex, France \\ *DRF/CENG, B.P. $85 \mathrm{X}, 38041$ Grenoble Cedex, France \\ **I.L.L., B.P. 156 X, 38042 Grenoble Cedex, France
}

Rësumé. - Nous avons obtenu ce que nous croyons être la première mise en évidence expérimentale de 1 'effet de Pendellösung en diffraction des neutrons par un cristal magnétique. L'échantillon, un monocristal de YIG, a été selectionné et taỉllé de façon que les défauts qu'il présente - des bandes de croissance, pour 1'essentiel $n$ 'aient pas d'influence sur la diffraction dans la géométrie que nous avons choisie. Nous avons mesuré le rapport de retournement $R$ ainsi que les pouvoírs réflecteurs $\rho_{+}$et $\rho$ - correspondant au deux polarisations du faisceau de neutrons, en fonction de la longueur d'onde. $R(\lambda)$ et $\rho_{+}(\lambda)$ présentent bien les oscillations prévues dans le cas d'un cristal parfait, mais avec une amplitude plus faible dont on peut rendre compte, par exemple, en utilisant la théorie statistique de la diffraction đéveloppée par Kato.

Abstract. - We have obtained what we believe to be the first experimental evidence, in neutron diffraction from a magnetic crystal, of the Pendellösung effect predicted by dynamical theory. We measured the flipping ratio, $R$, and the reflecting powers for both polarizations, $\rho_{+}$and $\rho_{-}$as a function of wavelength, from a carefully selected YIG single crystal, cut in a such a way that its main defects - growth bandsare inoperative in the chosen scattering geometry. Both $R(\lambda)$ and $p+(\lambda)$ present characteristic oscillating behaviours, related to those calculated for a perfect crystal. It is possible to account for the discrepancy between the experimental and calculated amplitudes of the oscillations using e.g. Kato's statistical theory of diffraction.

\title{
1. Introduction
}

An oscillatory behaviour of the integrated reflectivity is predicted by the dynamical theory when neutrons (or X-rays, or electrons) are Bragg-diffracted in transmission by a non-absorbing perfect crystal. This behaviour, which describes the coherent transfer of the flux of energy back and forth between the transmitted and the reflected beam directions, is analogous to the oscillations of energy from one to the other of two equal coupled pendulums, and was called by Ewald, in 1916, "Pendellösung" [1]. Pendellösung fringes have been investigated in many neutron diffraction experiments in nearly perfect non magnetic crystals $[2,3,4,5,6,7]$. Although theoretically predicted by different authors $[8,9,10,11,12,13]$ no observation of their magnetic counterpart has been reported, in spite of several attempts. This is not very surprising, since perfect enough magnetic crystal are not available. The present paper presents the way we overcome this difficulty, by arranging a geometrical situation where, for a given family of lattice planes, the sample behaves like a nearly perfect crystal, and reports the first observation of magnetic Pendellösung oscillations. The implications of this experiment are then discussed.

\section{A "perfect" sample in an imperfect crystal}

We choose to work on crystals of $\mathrm{Y}_{3} \mathrm{Fe}_{5} \mathrm{O}_{12}$ (YIG), on which a lot of accurate work was done and has provided interesting physics on the basis of heavy extinction cor-

\footnotetext{
+ present address: Physik-Department, Technische Universität Mïnchen, 8046 Garching,
} F.R.G. 
rections [14] . Flux-grown crystals of $\mathrm{Y}_{3} \mathrm{~F}_{5} \mathrm{O}_{12}$, though not exempt from defects, are among the magnetic-crystals featuring the best crystalline quality : regions of several $\mathrm{mm}^{3}$ can be obtained free from dislocations. As the other crystals grown by the same technique, they exhibit, as a rule, plane natural faces. An as-grown crystal is thus usually divided into different growth sectors, characterized by different sharply defined growth directions (fig.1). The dotted lines represent the contours of the crystal at different stages of growth. These contours are visible, sometimes optically and nearly always on X-ray topographs in such crystals, and are called "growth bands". These planar defects, nearly unavoidable, are the main

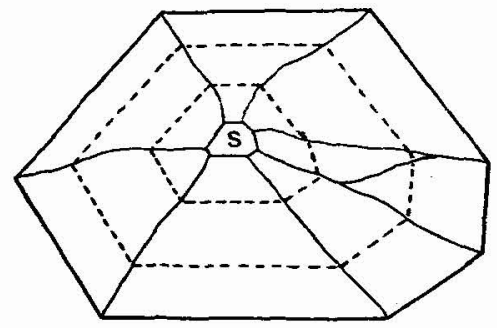

Fig. 1 - Schematic drawing showing the growth sectors in a flux grown crysta1.

The dotted lines indicate the contours of the crystal at different stages of growth.

inhomogeneities in flux-grown YIG samples. They arise from very slight changes in the lattice periodicity associated, e.g. with small local variations of the impurity content, and are created by fluctuations in the growth conditions.

An important point is that the atomic displacements around the fault must, by symmetry, be normal to the plane if boundary effects are neglected. Now any displacement field perpendicular to the diffraction vector $\overrightarrow{\mathrm{h}}$ has no influence from a diffraction point of view. It is thus possible to simulate a perfect crystal if $\overrightarrow{\mathrm{h}} \cdot \overrightarrow{\mathrm{f}}=0$, where $\overrightarrow{\mathrm{f}}$ is the growth band fault vector, normal to the growth band itself.

A $0.5 \mathrm{~mm}$ thick slice was thus cut in a YIG flux-grown crystal, normal to the [112] growth direction and near the corresponding (112) natural face, to be used in hho symmetrical Laue setting. The sample was carefully mechanically polished and chemically etched in order to remove surface damage. Its final thickness was $0.48 \mathrm{~mm}$ $\mathrm{X}$-rays topographs have shown, as expected, that its central part mainly consists of one single growth sector with growth bands parallel to the surface, hence not visible on the topograph (fig.2), where most of the contrasts are related to magnetic domains, and vanish when a magnetic field is applied along [11i] , the magnetic easy direction contained in the sample surface. An area $22.2 \mathrm{~m}$ in diameter was selected in the best region of the main growth sector (dotted lines on fig.2), i.e.

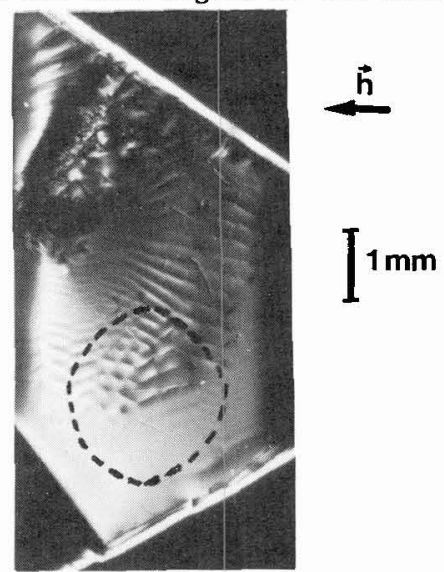

Fig. 2 - X-ray topograph of the YIG sample. MoKa, reflection $8 \overrightarrow{80}$. The dotted lines indicate the region selected to be illuminated by the neutron beam (see text).

that giving, for X-rays, the maximum anomalous transmission ("Borrmann") effect, a phenomenon which only occurs on perfect crysta1s [15]. The remainder of the crystal was masked by $1 \mathrm{~mm}$ thick cadmium and $0.4 \mathrm{~mm}$ thick gadolinium foils. 


\section{Dynamical and kinematical theory expectations}

Fig. 3 shows the integrated reflecting power in the Laue case, in the frame of dynamical theory, as a function of a dimensionless parameter proportional to the sample thickness $t$, called A by Zachariasen [16]:

$$
A=\frac{\pi t}{\Lambda}
$$

where $\Lambda$ is the extinction length, or Pendellösung period.

$$
\Lambda=\frac{\pi v_{c} \sqrt{\gamma_{0} \gamma_{h}}}{\lambda\left|\overrightarrow{F_{h}}\right|}
$$

$\gamma_{0}$ and $\gamma_{h}$ are the direction cosines of the incident and diffracted wave respectively, $\lambda^{\circ}$ is the wavelength of the neutrons, $v_{c}$ the volume of the unit cell and $F_{\vec{h}}$ the structure factor of the Bragg reflection we are concerned with. Of course $\sqrt{\gamma_{0} \gamma_{\vec{h}}}=\cos \theta_{B}$ in the symmetrical Laue case.

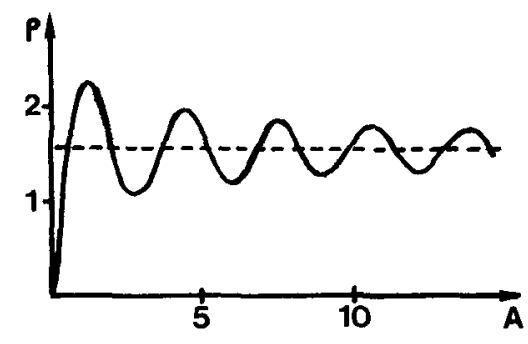

Fig.3 - The integrated reflecting power in the Lave case (transmission) as a function of $\mathrm{A}$.

Now, consider the simple case of a mixed (nuclear + magnetic) reflection from a single domain sample magnetized perpendicularly to the scattering plane. The Pendellösung periods $\Lambda_{+}$and $\Lambda_{-}$are different for "up" and "down" neutron polarizations, parallel and antiparallel to the magnetization, because the magnetic structure factor $F_{M}$ is added to or substracted from the nuclear one $F_{N}$. It is possible to scan the curve $\rho_{\vec{h}}(A)$ either by changing the thickness of the sample or, in a more convenient way from an experimental point of view, with constant thickness, by varying the wavelength. Fig.4a shows the two curves $\rho_{\vec{h}}^{t}$ and $\rho \overrightarrow{\vec{h}}$, as functions of $\lambda$, calculated for the $2 \frac{2}{0}$ reflection for a $0.48 \mathrm{~nm}$ thick perfect crystal of YIG, using the values of $F_{M}$ and $F_{N}$ derived in reference [14]. As the monochromatic beam intensity and the geometrical conditions for the diffraction change when $\lambda$ is changed, the flipping ratio $R(\lambda)$ (fig.4b) appears to be more reliable than $\rho \frac{\vec{h}}{h}(\lambda)$ and $\rho_{\bar{h}}(\lambda)$ separately.

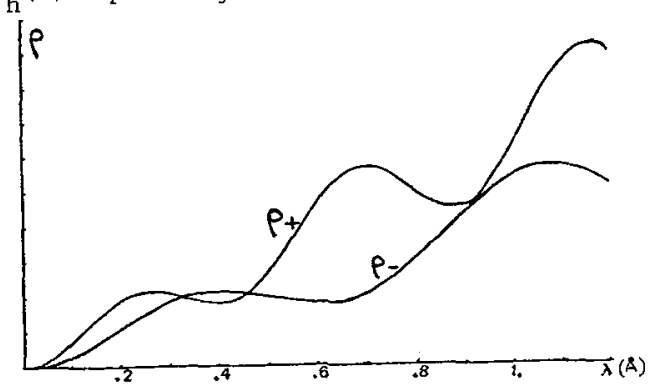

(a)

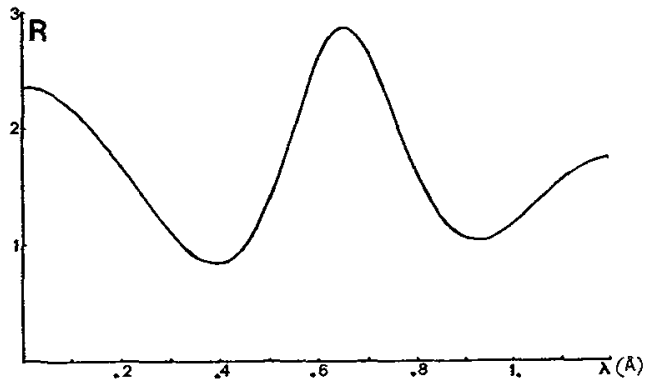

(b)

Fig. 4 - Curves calculated within the framework of dynamical theory, for the $2 \overline{2} 0$ reflection of a $0.48 \mathrm{~mm}$ thick YIG crystal a) reflecting powers $\rho_{+}$and $\rho_{-}$and b) flipping ratio, as a function of wavelength. 
The usual "kinematical" approximation $\left(R_{K}=\left|F_{+}\right|^{2} /\left|F_{-}\right|^{2}\right)$ corresponds to the zero thickness limit of the previous "dynamical" approach. The corresponding flipping ratio $R_{K}$ is independent of $\lambda$.

\section{Results and discussion}

The measurements were performed on the diffractometer D5 of ILL, using the wavelength range $0.42 \AA$ to $1.05 \AA$. The beam was polarized and monochromatized using the 111 reflection of a Cu2MnAl Heusler alloy crystal with mosaic spread $0.4 \circ$. The intensities were recorded at the maximum of the rocking curve. As our sample presents nearly no mosaíc distribution (a few seconds of arc), the flipping ratio is not expected to vary over the rocking curve, contrary to what occurs when investigating samples with an important secondary extinction [17, 18]. The flipping ratios $R$ we obtained were thus compared with the calculations of the previous sections, based on integrated intensities, just modified to take into account the experimental polarization ratio and flipping efficiency; the FWHM of the rocking curve is nearly constant through all the wavelength range we are concerned with.

A $1 \mathrm{~mm}$ thick foil of erbium was used as $\lambda / 2$ filter in the range $0.7-0.94 \AA$, taking advantage of the neutron absorption peak of this element located around $0.4 \AA$, while a plutonium filter was used when carrying out measurements at $\lambda=1.05 \AA$

The $\lambda / 2$ beam contaminates our measurements through the $4 \overline{4} 0$ reflection of YIG. In order to correct for this extra intensity, 1) we measured the $(\lambda / 2) 2 \overline{2} 0$ reflection, for both polarizations, by setting the sample to diffract the main beam $(\lambda)$ on the forbidden $1 \overline{1} 0$ reflection, 2) we deduced the polarization ratio of the $(\lambda / 2)$ beam from these measurements, 3) we measured, as a function of $\lambda$, the $4 \overline{4} 0$ reflection, and deduced the ratio $\alpha=I(4 \overline{4} 0) / I(2 \overline{2} 0)$ for both polarizations. 4) we noted that the monochromatic beams scattered are obtained, in the different experiments, from different monochromator reflections -111 and 222 -, and checked that this fact does not significantly modify the $\alpha$-ratios we have to use.

Corrections accounting for incomplete neutron absorption by the Cd + Gd foils covering the less perfect part of the crystal were also performed for the points corresponding to wavelength smaller than $0.47 \AA$. Last it was checked, restricting the analysis to $|\vec{h}|$ smaller than $8.7 \mathrm{a}^{*}$, that no Renninger effect (simultaneous reflections) occurs in the scattering plane in our geometry.

Fig. 5 shows the measured flipping ratios $R$ as well as the calculated curve. The experimental reflectivities $\phi_{+}$and $\rho_{-}$are shown on fig. 6 ; to derive them we used the counts recorded by the monitor at each wavelength, corrected for the monitor efficiency and the $(\lambda / 2)$ radiation. A first remark is that the experimental $R$ curve of fig. 5 oscillates as a function of wavelength, which is quite unusual in polarized neutron diffraction. This oscillation is in qualitative agreement with the calculated curve, but its amplitude is about twice smaller. This indicates that the sample does not behave exactly like a perfect crystal, probably simply because it is not perfect enough !

A crude model, which assumes a fraction of the sample to behave kinematically, does not account for experimental results. More elaborate theories like Kato's [19] have to be used and allow, by adjusting the values of the "static Debye-Waller factor" $E$ and of the coherence length $\tau$, a good fit of the obtained curve. This approach could then lead to a quantitative determination of the ratio $F_{N} / F_{M}$. We checked that both the amplitude of the oscillations and the positions of the maxima and minima depend upon $\mathrm{E}$ and $\tau$.

The eurves of Fig. 6 are also in qualitative agreement with calculated ones (Fig. 4a). In particular $p_{+}$presents a maximum and a minimum in the wavelength range we are concerned with, like the perfect crystal corresponding curve. The uncertainties involved in the treatment of uncorrected measurements preclude a very precise determination of scattering parameters, but an encouraging preliminary result was nevertheless obtained by the use of the "wavelength-ratio" method, and is discussed in another paper of this conference $[20]$. 


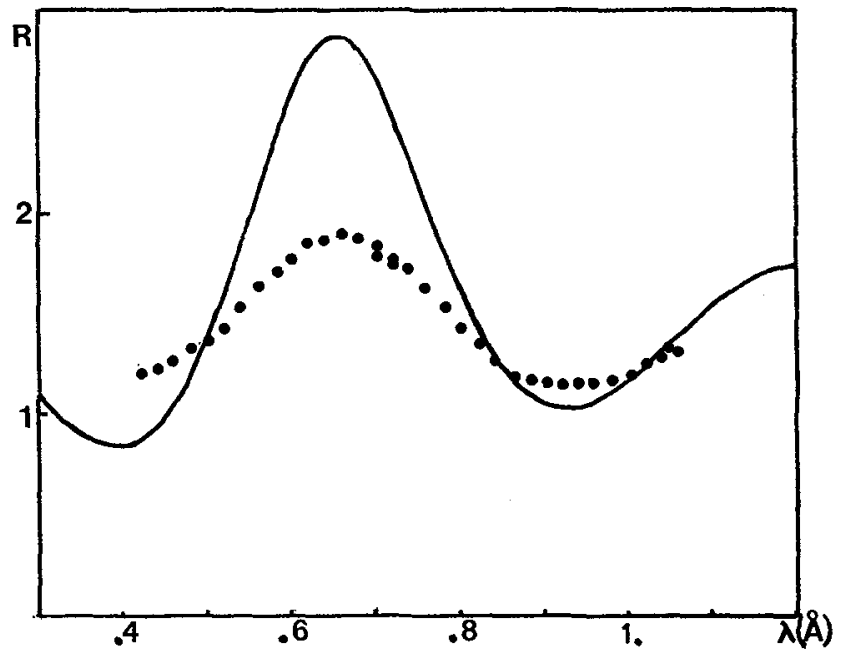

Fig. 5 - Flipping ratio, measured (points) and calculated for a perfect crystal, as a function of neutron wavelength. $2 \overline{2} 0$ reflection, $0.48 \mathrm{~mm}$ thick YIG crystal

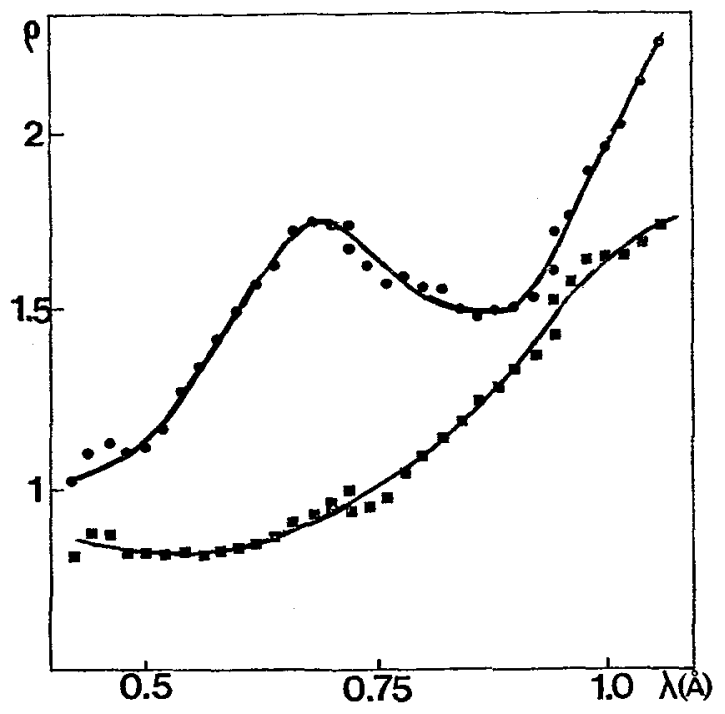

Fig. 6 - Reflecting powers $\rho_{+}(\bullet)$ and $\rho_{-}(\boldsymbol{\bullet})$, in arbitary units, measured as a function of wavelength for the 220 reflection of a $0.48 \mathrm{~mm}$ thick YIG crystal. 


\section{Conclusion}

We have performed what we think is the first observation of magnetic Pendellösung effect. This experiment open the way to a lot of experimental work on diffraction by nearly perfect magnetic crystals.

Acknow1edgements

We are grateful to W. Graeff for kindly computing possible simultaneous reflections in the scattering plane for our geometry.

\section{References}

[1] Ewald, P.P., Acta Cryst. A.25 (1969) 103.

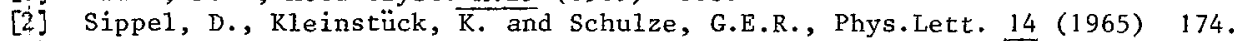

[3] Shul1, C.G., Phys.Rev.Lett., 21 (1968) 1585.

[4] Kikuta, S., Kohra, K., Minakawa, N. and Doi, K., J.Phys.Soc.Japan, 31 (1971) 954.

[5] Malgrange, C., Pétroff, J.F., Sauvage, M., Zarka, A. and Englander, M., Phil.Mag. 33 (1976) 743.

[6] Somenkov, v.A.S., Shilstein, S.Sh., Belova, N.E., and Utemisov, K., Solid State Comm. 25 (1978) 593.

[7] Tomimitsu, H. and Zeyen, C., Japan. J.Appl.Phys. 17 (1978) 591.

[8] Stassis, C. and Oberteuffer J.A., Phys.Rev. B10 (1974) 5192.

[9] Sivardière, J., Acta Cryst. A31 (1975) 340.

[10] Schmidt, H.H. and Deime1, P., Phys.Stat.Sol. B73 (1976) 87, and other references therein.

[11] Mendiratta, S.K. and Blume, M., Phys.Rev. B74 (1976) 144.

[12] Belyakov, V.A. and Bokun, R.CH., Soviet Phys.Sol.St. 18 (1976) 1399

[13] Guigay, J.P. and Schlenker, M., in "Neutron Interferometry", edited by U.Bonse and H. Rauch, Clarendon Press, Oxford (1979), p. 135.

[14] Bonnet, M., Delapalme, A., Fuess, H. and Becker, P., J.Phys.Chem.Solids, 40 (1979) 863.

[15] See, for instance, Batterman, B.W. and Cole, M., Rev. of Modern Physics 36 (1964) 681 .

[16] Zachariasen, W.H., "Theory of X-ray diffraction in crystals" (1967), Dover, New York.

[17] Nathans, A., Shu11, C.G. Shirane G. and Andresen A., J.Phys.Chem.Solids

[18 (1959) 138.

[18] Dobrzynski, L., Nukleonika 25 (1980) 807.

[19] Kato, N. Acta Cryst. A36 (1980) 763 and 770.

[20] Guigay, J.P., Schlenker M., and Baruchel J., paper presented at this Conference 